Rev. Adm. Saúde - Vol. 18, № 70, jan. - mar. 2018

http://dx.doi.org/10.23973/ras.70.81

ARTIGO ORIGINAL

\title{
Proposta de redução de estoque excedente de um complexo hospitalar de alta complexidade
}

Proposal of a Surplus Stock Reduction in a High Complexity Hospital

\author{
Patricia Mora Pereira ${ }^{1}$, Roberta Selingardi ${ }^{2}$
}

1. Enfermeira, mestra em ciências da saúde, especialista em administração em saúde. Aprimoranda do PROAHSA, Hospital das Clínicas da Faculdade de Medicina da USP.

2. Biomédica, especialista em administração em saúde. Aprimoranda do PROAHSA, Hospital das Clínicas da Faculdade de Medicina da USP.

\section{RESUMO}

Estima-se que de 35 a $45 \%$ do orçamento total do hospital é associado a administração de materiais, ou seja, quase $50 \%$ dos recursos financeiros são destinados aos materiais e medicamentos da organização, sendo a gestão desse estoque um processo importante para uma gestão estratégica voltada para a redução de custos. Estoques sem consumo e sem rotatividade na organização de saúde são considerados excedentes, pois permanecem por um longo tempo na organização, não possuem giro de estoque e não geram lucro e nem retorno ao investimento realizado, podendo se transformar em perda por obsolescência ou perecibilidade. Desta forma, este estudo teve como principal objetivo identificar se existe estoque excedente de materiais e medicamentos em um complexo hospitalar de alta complexidade e propor planos de ação com o intuito de minimizar possíveis perdas por perecibilidade. Ao considerar o objeto de investigação, optou-se por realizar um estudo descritivo, exploratório com a utilização da abordagem quantitativa e qualitativa de pesquisa. Em um primeiro momento, foram coletados dados sobre a movimentação dos insumos no estoque, quantificando-os e qualificando-os por meio da curva $A B C$ para os fins pretendidos, sendo esta a abordagem quantitativa. Do mesmo modo, em um segundo momento, também foram levantadas informações referentes a gestão de estoque, por meio de relatos dos colaboradores e de um estudo 
observacional com o intuito de mapear os gaps do processo, sendo esta segunda abordagem definida como qualitativa. Os resultados mostraram que 710 insumos dos grupos medicamento e material médico hospitalar possuíam estoque excedente, entretanto, apenas 80 insumos foram classificados como A, ou seja, correspondiam a $80 \%$ do valor total de estoque destes grupos. Assim, foram propostos planos de ação para os 80 insumos visando a sua redução imediata. Além disso, também foram identificados, por meio da ferramenta fluxograma, os principais gaps do processo de gestão de estoque com posterior sugestão de melhoria para o processo e propostas de ação para prevenir a formação futura de estoque excedente. A formação de estoque excedente está atrelada a muitas variáveis, entretanto, embora seja uma gestão de difícil controle, o complexo hospitalar preza pela excelência e busca a melhoria contínua dos seus processos, de forma que têm interferido de maneira significativa nessas variáveis, por meio do uso de ferramentas e sistemas de gestão de estoque, reduzindo assim seus custos e proporcionando uma assistência ao paciente com mais segurança e qualidade.

Palavras-chave: gestão em saúde; estoque estratégico; gestão de recursos.

\section{ABSTRACT}

It is estimated that 35 to $45 \%$ of a hospital budget is associated to materials administration, in other words, almost $50 \%$ of the financial resources are destined to hospital material and medicine drugs of the organization, making the administration of this stock an important process to a strategic cost reduction management. Stocks without consumption and turnover are considered surplus, because they stay for a long time in the organization, do not have inventory turnover and do not give profit or payback to their investment, being able to become a loss of supplies due to obsolescence or perishability. Thus, this study had as primary objective to identify if there was surplus stock of hospital materials and medicine drugs in a high complexity public hospital and propose action plans in order to minimize possible losses of supplies due to perishability. When considering the investigation object, it was chosen to make a descriptive and exploratory study, using the quantitative and qualitative research approach. In a first moment, there was collected data about the stock's supplies movement, quantifying and qualifying it through the $A B C$ curve method for the intended purposes, being this the quantitative approach. Likewise, in a second moment, there was also gathered information regarding the stock management, through employee's reports and an observational study with the aim to map the process gaps, being this second the qualitative approach. The results show that 710 supplies from the group of medicine drugs and hospital material had surplus stocks, meanwhile, only 80 supplies were classified as $A$, corresponding to $80 \%$ of the total stock value of these groups. With that, there were proposed action plans for each of the 80 supplies aiming to its immediate reduction. Furthermore, it was also identified, through the Flow Chart tool, the main gaps of the stock management process in this hospital complex with subsequent suggestions for improvement to the process and proposals for action to prevent the future formation of surplus stock. The formation of surplus stock is tied to many variables. However, even though it is a difficult to administrate 
management, this hospital complex values excellence and seeks continuous improvements of its process, in a way that it had interfered significantly on these variables, through the use of stock management systems and tools, reducing their costs and providing to the patient a safer and quality health care.

Keywords: health management, strategic stockpile, resources management.

\section{INTRODUÇÃO}

Nos últimos anos, as organizações de saúde têm sofrido profundas modificações, especialmente, as organizações de saúde de administração pública. Com a diminuição do número de beneficiários de planos de saúde privado, aumentou-se a demanda e a busca pela universalização do acesso às organizações de saúde públicas, entretanto, os custos em saúde não se mantiveram estáveis, pelo contrário, continuaram a expandir com a introdução de novas tecnologias e novas opções terapêuticas, gerando a necessidade de uma gestão mais estratégica ${ }^{1}$.

Um dos processos que podem ser avaliados e impactar de forma significativa na redução dos custos é a administração de materiais, especificamente, o gerenciamento de estoques. Estima-se que de 35 a $45 \%$ do orçamento total do hospital é associado a administração de materiais, ou seja, quase $50 \%$ dos recursos financeiros são destinados aos materiais hospitalares e medicamentos da organização².

A principal finalidade da administração de materiais é prover os recursos necessários para uma assistência de qualidade, em quantidade de materiais hospitalares e medicamentos suficientes, no tempo correto e com o menor custo para o serviço público de saúde; sendo o gerenciamento do estoque uma etapa relevante da cadeia de suprimentos e do sistema de produção ${ }^{3}$.

Estoque, conforme definição de Silva et aA é o acúmulo de suprimentos, principalmente, materiais hospitalares e medicamentos que são adquiridos e armazenados para utilização futura em uma organização de saúde. O estoque permite a geração de lucro para a organização quando ocorre seu giro de estoque, ou seja, o material e/ou medicamento é consumido pelo paciente e o capital empregado para a sua aquisição é retornado de volta para a organização ${ }^{3}$.

A necessidade de formação de um estoque acima do necessário para atender uma determinada demanda (estoques de segurança) surge devido as incertezas que existem entre as fases de um processo de transformação, ou seja, para manter os níveis de prestação de serviço adequados, atender as demandas diversas e sazonais que aparecem no complexo hospitalar e não 
sofrer impacto das taxas de incerteza como o atraso nas entregas de suprimentos pelos fornecedores ${ }^{5}$.

Em geral, as organizações de saúde preferem possuir um estoque acima do necessário para atendar a sua demanda, elevando o custo de armazenagem, do que possuir o custo da falta de medicamento, que se for um insumo crítico para a atividade-fim da organização (insumos classificados em Z pela classificação $X Y Z$ ) pode colocar em risco à vida dos pacientes, gerar processos judiciais e indenizações, além de denegrir a imagem da organização de saúde e gerar impactos negativos para a sua reputação ${ }^{6}$.

No entanto, é observado, muitas vezes, a formação de um estoque excedente, ou seja, um estoque com quantidade de materiais hospitalares e medicamentos acima do estoque de segurança necessário para uma assistência de qualidade. Com isso, esses estoques costumam não apresentar consumo e nem rotatividade, sendo considerados excedentes, uma vez que permanecem por um longo tempo na organização, não possuem giro de estoque e não geram lucro e nem retorno ao investimento realizado, podendo, muitas vezes, se transformar em perda por obsolescência ou perecibilidade. Além disso, estoques excedentes elevam as quantias de capital imobilizado, requerem maiores espaços para o armazenamento dos suprimentos, elevam as despesas financeiras com recursos humanos, manuseio e movimentação interna e gera desequilíbrio no balanço patrimonial com a diminuição dos ativos circulantes, o que impacta diretamente na liquidez da organização impedindo que a organização consiga alocar recursos em áreas estratégicas do hospital ${ }^{3}$.

Desta forma, este estudo buscou identificar se existe estoque excedente de materiais hospitalares e medicamentos em um complexo hospitalar de alta complexidade, visando diminuir possíveis perdas por obsolescência ou perecibilidade dos estoques que estejam sem giro por um longo período. Além disso, buscou-se também propor ações que possam auxiliar no giro deste estoque gerando um retorno ao investimento realizado para a aquisição dos suprimentos, bem como, propor medidas para evitar a formação de estoque excedente, de forma a diminuir os custos operacionais de forma sustentável e permitir que os recursos financeiros escassos sejam alocados em áreas estratégicas da organização.

\section{OBJETIVOS}

O objetivo geral deste estudo foi verificar a existência de estoque excedente (estoque com cobertura acima de 90 dias) de materiais hospitalares e medicamentos em um complexo hospitalar de alta complexidade e propor planos de ação com o intuito de minimizar possíveis perdas por perecibilidade.

Os objetivos específicos foram descrever e mapear as lacunas (gaps) do processo de gestão de estoque; identificar os insumos, classificados nos grupos medicamento ou material médico hospitalar, que possuem estoque excedente devido à ausência de rotatividade; propor planos de ação visando a diminuição do estoque excedente por meio de critério preestabelecido e propor 
planos de ação para prevenir a formação futura de estoque excedente no complexo hospitalar de alta complexidade.

\section{PROCEDIMENTOS METODOLÓGICOS}

Ao considerar as características do objeto de investigação do presente estudo, optou-se por realizar um estudo descritivo, exploratório com a utilização da abordagem quantitativa e qualitativa de pesquisa.

Em um primeiro momento, foram coletados dados sobre a movimentação dos insumos/itens no estoque do complexo hospitalar de alta complexidade, quantificando-os e qualificando-os, por meio da curva $A B C$, para os fins pretendidos, sendo esta a abordagem quantitativa. Do mesmo modo, em um segundo momento, também foram levantadas informações referentes a gestão de estoque, por meio de relatos dos colaboradores e de um estudo observacional, sendo esta segunda abordagem definida como qualitativa.

Os dados desse estudo são primários e foram coletados em dezembro de 2016, por meio de um estudo prático-observacional no setor de logística e gestão de suprimentos do complexo hospitalar; relato dos colaboradores que atuam nesse setor e disponibilização de documentos internos, bem como, da "Planilha de Fechamento Mensal" com os dados referentes ao consumo e ao estoque dos itens ativos no complexo hospitalar no período de janeiro a dezembro de 2016.

Os itens que foram analisados nesse estudo possuíram como critério de inclusão: serem classificados pelo setor de suprimentos como pertencentes aos grupos material médico hospitalar ou medicamentos; possuírem rotatividade ausente (consumo igual a zero) no período de janeiro de 2016 a dezembro de 2016; possuírem estoque no mês de dezembro de 2016 e estarem elencados na "Planilha de Fechamento Mensal" gerenciada pelo setor de suprimentos do complexo hospitalar. Foi definido como critério de inclusão os insumos pertencentes aos grupos medicamento e material médico hospitalar, devido ao maior impacto que esses insumos possuem na qualidade da assistência direta ao paciente.

Como critérios de exclusão podemos citar: serem classificados pelo setor de suprimentos como pertencentes aos grupos EPI (equipamento de proteção individual), laboratório, materiais diversos, nutrição ou OPME (órteses, próteses e materiais especiais); possuírem consumo em qualquer mês dentro do período de janeiro de 2016 a dezembro de 2016 e não possuírem estoque no mês de dezembro de 2016.

Para a análise dos dados, obtidos por meio da "Planilha de Fechamento Mensal", foram estabelecidas as seguintes etapas sequenciais:

- Identificação de todos os insumos/itens que possuíram rotatividade ausente (consumo igual a zero) no período de janeiro de 2016 a dezembro de 2016; 
- Identificação de todos os insumos/itens, anteriormente classificados com rotatividade ausente, que possuíram estoque no mês de dezembro de 2016;

- Identificação dos insumos, pertencentes aos grupos medicamento ou material médico hospitalar, que possuíam rotatividade ausente e estoque excedente;

- Construção da curva $A B C$ de valor dos insumos pertencentes aos grupos material médico hospitalar e medicamentos com rotatividade ausente e estoque excedente;

- Definição dos insumos, pertencentes aos grupos medicamentos ou material médico hospitalar, que foram classificados como A, por meio da curva $A B C$ de valor realizada na etapa IV.

Após uma análise minuciosa desses insumos, classificados nas etapas descritas acima, foram elaborados planos de ação visando a sua redução no estoque. Além disso, também foi realizado uma análise do processo de gestão de estoques com a descrição do processo e o mapeamento das lacunas (gaps) por meio da ferramenta de qualidade fluxograma; foram destacadas as oportunidades de melhoria do processo, com posterior sugestão de melhorias e, por fim, foram propostos planos de ação para prevenir a formação futura de estoque excedente no complexo hospitalar.

\section{RESULTADOS}

Conforme descrito no tópico procedimentos metodológicos deste estudo, os primeiros passos foram a identificação dos insumos/itens, pertencentes aos grupos de material médico hospitalar e medicamentos, que possuíam estoque excedente, ou seja, estoque com cobertura acima de 90 dias (estoque máximo desejável no complexo hospitalar); rotatividade ausente (consumo igual a zero) no período de janeiro de 2016 a dezembro de 2016 e possuía estoque presente no mês de dezembro de 2016.

Após a definição inicial de todos os insumos/itens que possuíam rotatividade ausente no ano de 2016 e estoque presente em dezembro de 2016, realizou-se a metodologia da curva $\mathrm{ABC}$ de valor. A primeira etapa para a elaboração da curva $A B C$ de valor consiste em ter duas séries de dados: a quantidade de insumos/itens e custo médio unitário. Assim, os insumos/itens classificados em A correspondem a $80 \%$ do valor total (em reais) de estoque, os insumos/itens classificados em B correspondem a $15 \%$ do valor total de estoque e os classificados em C correspondem a 5\% do valor total de estoque.

Desta forma, foram elencados os insumos pertencentes aos grupos material médico hospitalar e medicamentos que possuíam rotatividade ausente e estoque em dezembro de 2016, chegando a um total de 710 insumos. Esses 710 insumos correspondem a um valor total de estoque de $R \$ 1.390 .355,42$. 
Posterior a definição dos insumos, esses foram classificados pela metodologia da curva $A B C$ de valor, chegando em um total de 80 insumos classificados como A, ou seja, $11 \%$ dos insumos analisados correspondem a $80 \%$ do valor de estoque total dos grupos material médico hospitalar e medicamentos, conforme podemos observar pela Figura 1.

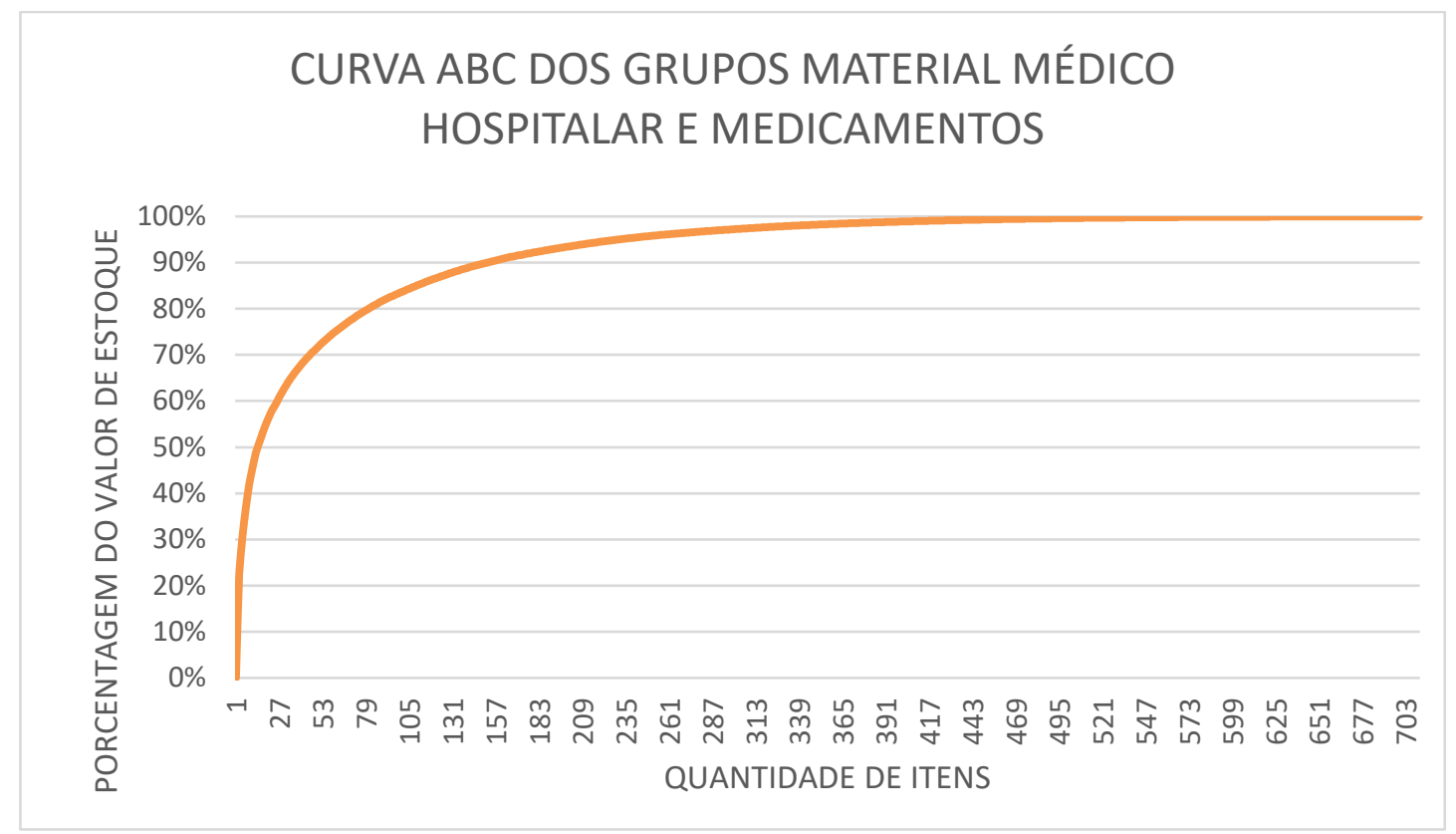

Figura 1. Curva $A B C$ de valor dos grupos material médico hospitalar e medicamentos

Devido ao tempo limitado do estudo e considerando a literatura que aponta que os insumos que devem receber atenção prioritária dos gestores são os classificados como A, por possuírem $80 \%$ do valor total de insumos analisados, foi definido que seriam analisados de forma minuciosa os 80 itens pertencentes a classificação $A$ dos grupos material médico hospitalar e medicamentos. Esta análise consistiu em analisar os seguintes pontos: institutos, pertencentes ao complexo hospitalar, que também fazem o uso deste insumo e estão com cobertura para o uso abaixo de 60 dias, o que permitia uma transferência imediata e insumos que não possuem consumo em nenhum instituto e estão com estoque sendo necessário propor formas para a sua redução imediata.

A principal proposta de ação para a redução imediata do estoque excedente é a transferência dos suprimentos que estão com estoque excedente para outros institutos do complexo hospitalar que fazem o uso destes insumos e estão com pouco estoque, ou seja, possuem consumo atual e a sua cobertura está abaixo de 60 dias e estes necessitam realizar um pedido de reposição de estoque. Antes de realizar a transferência imediata, uma atenção que precisa ser dada aos insumos é se estes não ultrapassaram o seu prazo de validade, entretanto, como o Centro de Distribuição não recebe insumos com prazo de validade em 
um tempo inferior a 18 meses, acredita-se que os insumos elencados não terão ultrapassado o seu prazo de validade.

Com isso, dos 80 insumos pertencentes aos grupos medicamentos e material médico hospitalar e classificados como A na curva ABC de valor, 20 insumos eram passíveis de redução imediata por meio da transferência entre institutos. Esses 20 insumos correspondem a $25 \%$ do total de insumos elencados, porém correspondem a $32,25 \%$ do valor total dos 80 insumos, ou seja, $R \$ 358.739,56$.

Em relação aos 60 insumos restantes que não possuem consumo em nenhum instituto, uma proposta de ação para a sua redução imediata é a avaliação junto a diretoria clínica do complexo hospitalar e a alta liderança de cada instituto se estes insumos podem ser substitutos de insumos que estão com cobertura baixa ou possuem giro de estoque alto. Insumos substitutos são os insumos classificados em Y na classificação de criticidade XYZ e correspondem a insumos que podem ser substituídos por insumos de ação equivalente com relativa facilidade. Uma vez avaliado estes 60 insumos e definidos que esses podem ser substitutos, deve-se padronizá-los, temporariamente, nos institutos por meio da Comissão de Padronização de Materiais e Medicamentos e realizar a conscientização da equipe médica e multiprofissional para que estes insumos tenham o seu uso estimulado.

Uma limitação que esta proposta de ação pode possuir é os insumos não serem substitutos a nenhum outro insumo atualmente utilizado ou a equipe médica negar a sua utilização, uma vez que esta avalia que este insumo não possui evidências científicas sobre sua eficácia e segurança na assistência ao paciente. Caso ocorra a primeira situação sugere-se a transferência/doação para outros serviços de saúde do Sistema Único de Saúde que o utilizam na atenção secundária e/ou terciária. Já na segunda situação, sugere-se aceitar a perda, porém monitorar para que estes insumos não sejam comprados futuramente para uso no complexo hospitalar.

\section{DISCUSSÃO}

Através da análise da descrição do processo de gestão de estoque, podemos apontar sugestões de melhoria.

A primeira sugestão de melhoria é em relação ao processo de compra de suprimentos que deve ser planejada com métodos mais eficazes para que não ocorra a compra emergencial, a qual aumenta o custo para a organização de saúde, visto que os suprimentos adquiridos fora da modalidade de licitação têm maior custo, além dos custos atrelados a falta do suprimento que podem colocar em risco à vida do paciente e aumentar as chances de indenizações e processos judiciais.

Embora a compra no complexo hospitalar atualmente seja planejada com base na demanda observada na série histórica, observa-se algumas limitações neste método de previsão. Uma limitação são os empréstimos realizados entre os institutos que aparecem no sistema de gestão como saída para consumo e não 
como empréstimo, demonstrando um consumo não fidedigno com a realidade. Outra limitação é que este método não indica flutuações de demanda que não sejam sazonais, dificultando a previsão e gerando uma reação lenta às mudanças no comportamento da demanda.

Outra sugestão é a melhoria na avaliação dos fornecedores. Foi constatado neste estudo que algumas entregas estão sem conformidade, sendo o principal motivo o atraso no tempo de entrega acordado, o que aumenta o lead time e coloca em risco a continuidade da assistência prestada ao paciente. É necessário avaliar continuamente estes fornecedores de maneira a identificar eventuais causas para a não conformidade, bem como propor planos de ação junto com os mesmos de maneira a evitar a recorrência dessas ações não conformes. Podemos citar como exemplo de dimensões que podem ser avaliadas para qualificar os fornecedores a disponibilidade de suprimentos para pronto-atendimento; a rapidez nas entregas; a pontualidade no cumprimento do prazo das entregas acordadas e a qualidade das entregas realizadas.

Além disso, este estudo propõe também propostas de ação específicas para prevenir a formação futura de estoque excedente no complexo hospitalar. As propostas de ação definidas foram: estreitamento da relação com os fornecedores com o intuito de realizar negociações com lotes econômicos menores; avaliação frequente dos fornecedores com o intuito de propor planos de ação visando a diminuição do lead time, podendo acordar entregas periódicas sem que ocorra atrasos e descontinuidade da assistência prestada ao paciente, buscando assim manter na instituição estoques mais enxutos; classificação de todos os insumos pela curva $A B C$ de valor e pela classificação $\mathrm{XYZ}$ de criticidade, assegurando uma atenção diferenciada para cada combinação de maneira que os insumos $A Z$, ou seja, os insumos de maior custo e com alta criticidade possuam uma atenção prioritária; elaboração de um Procedimento Operacional Padrão (POP) para a alteração da padronização dos suprimentos, com o intuito de verificar se existe estoque do insumo anteriormente padronizado no complexo hospitalar antes de descontinuar o uso do mesmo, evitando assim a formação de estoque excedente por ausência de consumo; inserir alertas no sistema de gestão de estoque para indicar quando a cobertura estiver superior a 90 dias ou quando o insumo não possuir nenhum consumo em um período mínimo de 6 meses; treinamentos com os gestores das áreas assistenciais que fazem o pedido de compra com o intuito de conscientizá-los sobre os custos da falta ou do excesso de suprimentos na organização; definir uma pessoa responsável para analisar as flutuações no comportamento da demanda, de maneira que seja possível prever com antecedência quedas no consumo; monitoramento do consumo dos insumos requeridos pelas áreas que sejam classificados na curva $A B C$ de valor como $A$, ou seja, possuem maior custo para a organização; realização de inventários rotativos e gestão visual com maior periodicidade com o intuito de corrigir possíveis falhas nos dados gerados pelos sistemas de gestão de estoque.

\section{CONCLUSÃO}


Por meio deste estudo foi possível observar que embora a metodologia da classificação ABC ou classificação de pareto aponte que, aproximadamente, $20 \%$ dos insumos correspondem a $80 \%$ dos custos totais, os insumos com estoque excedente dos grupos medicamento e material médico hospitalar desse complexo hospitalar possuem valores de custo mais alto. Desta forma, dos 710 insumos totais destes grupos que foram classificados como estoque excedente, $11 \%$ ou 80 insumos correspondiam a $80 \%$ dos seus custos totais, sendo necessário que estes insumos recebam atenção prioritária devido ao seu alto custo para a organização.

A formação de estoque excedente está atrelada a muitas variáveis como, por exemplo, atraso da entrega por parte dos fornecedores; classificação de grande parte dos insumos, pelos gestores das áreas assistenciais, como sendo insumo crítico; alteração da padronização dos materiais e medicamentos sem conferência prévia sobre seu estoque; demora no processo de compra por licitação, entre outros fatores que dificultam a atuação dos gestores que realizam o planejamento e a gestão de estoque.

Assim, a gestão de estoque é uma gestão de difícil controle, entretanto, esse complexo hospitalar preza pela excelência e busca a melhoria contínua dos seus processos, de forma que têm interferido de maneira significativa nessas variáveis, por meio do uso de ferramentas e sistemas de gestão de estoque, reduzindo assim seus custos e proporcionando uma assistência ao paciente com mais segurança e qualidade.

Devido a identificação da oportunidade de melhoria ter ocorrido no início de 2017, esta proposta de ação já foi apresentada para a alta liderança do complexo hospitalar e para os responsáveis pela gestão de suprimentos e do estoque, os quais já adotaram algumas medidas propostas neste estudo.

Desta forma, conclui-se que as ações estratégicas para a melhoria da gestão do estoque não devem ser realizadas de forma verticalizada, mas com a participação de todas as áreas do hospital, sejam elas assistenciais, de apoio ou gerenciais, bem como com a participação de todos os envolvidos na cadeia de suprimentos. Com a compreensão da importância da gestão de estoque para a organização e com a participação de todos os envolvidos no processo de logística e gestão de estoque na elaboração de estratégias, é possível alcançar resultados melhores, engajar os envolvidos e prover uma assistência com mais qualidade e segurança ao paciente.

\section{REFERÊNCIAS}

1. Pena FPM, Malik AM, Viana FM. Gestão estratégica em saúde. In: Vecina Neto G, Malik AM. Gestão em saúde. Rio de Janeiro: Guanabara Koogan, 2016. cap. 8, p. 113-129. 
2. Reinhardt Filho W. Gestão de suprimentos e medicamentos. In: Vecina Neto G, Malik AM. Gestão em saúde. Rio de Janeiro: Guanabara Koogan, 2016. cap. 15 , p. $200-211$.

3. Barbieri JC, Machline C. Logística Hospitalar: teoria e prática. São Paulo: Saraiva, 2009.

4. Silva RB, et al. Logística em organizações de saúde. Rio de Janeiro: Editora FGV, 2010.

5. Corrêa HL, Corrêa CA. Administração de produção e operações: manufatura e serviços: uma abordagem estratégica. São Paulo: Atlas, 2011.

6. Wanke P. Tendências da gestão de estoques em organizações de saúde. ILOS - Especialistas em logística e supply chain. Brasil, 2004. [citado 27 maio 2017] Disponível em: <http://www.ilos.com.br/web/tendencias-dagestao-de-estoques-em-organizacoes-de-saude>.

Recebido: 16 de janeiro de 2018. Publicado: 20 de fevereiro de 2018

Correspondência: Patricia Mora Pereira. E-mail: patricia.mora89@gmail.com

Conflito de Interesses: os autores declararam não haver conflito de interesses.

(C) This is an Open Access article distributed under the terms of the Creative Commons Attribution License, which permits unrestricted use, distribution, and reproduction in any medium, provided the original work is properly cited 
